\title{
Semi-analytical modelling of linear scan voltammetric responses for soluble- insoluble system: The case of metal deposition
}

\author{
Imene Atek $^{\mathrm{a}, \mathrm{b}}$, Sunny Maye ${ }^{\mathrm{b}}$, Hubert H. Girault ${ }^{\mathrm{b}}$, Abed M. Affoune ${ }^{\mathrm{a}, *}$, Pekka Peljo ${ }^{\mathrm{b}, *}$ \\ ${ }^{a}$ Laboratoire d'Analyses Industrielles et Génie des Matériaux, Département de Génie des Procédés, Faculté des Sciences et de la Technologie, Université 8 Mai 1945 Guelma, \\ BP 401, Guelma 24000, Algeria \\ ${ }^{\mathbf{b}}$ Laboratoire d'Electrochimie Physique et Analytique, École Polytechnique Fédérale de Lausanne, EPFL Valais Wallis, Rue de l'Industrie 17, Case Postale 440, CH-1951 \\ Sion, Switzerland
}

\section{A R T I C L E I N F O}

\section{Keywords:}

Modelling

Metal deposition

Soluble-insoluble system

Linear sweep voltammetry

\begin{abstract}
A B S T R A C T
The absence of general theoretical models describing linear sweep voltammetry (LSV) or cyclic voltammetry (CV) responses for soluble-insoluble systems such as one-step electrodeposition reactions under quasi-reversible condition makes it difficult to extract quantitative kinetic information from experimental voltammograms. In this work, a semi-analytical method for modelling LSV responses for one-step electrodeposition process is described, for a case where instantaneous nucleation takes place, such as metal deposition on same metal. Voltammetric peaks were analyzed following variation of both dimensionless rate constants and charge transfer coefficients in a broad range. Therefore, kinetic curves for electron transfer processes were established and fitted perfectly by sigmoidal Boltzmann function and linear models. With these models, LSV or CV experimental data can be used to measure electrodeposition reactions kinetics whatever the degree of reversibility. The $\mathrm{Cu}(\mathrm{I}) / \mathrm{Cu}(0)$ couple in acetonitrile was selected as an experimental example. The model developed in this work predicts accurately the current response for $\mathrm{Cu}$ electrodeposition reaction and an excellent experiment-theory agreement was found.
\end{abstract}

\section{Introduction}

Over the years within the field of electrochemistry, many efforts have been devoted to modelling and simulation techniques to help to understand electroanalytical experiments [1-10]. Linear sweep voltammetry (LSV) and cyclic voltammetry (CV) are well-known electrochemical techniques which have played an important role to obtain a clear view about the kinetics, thermodynamics and mechanisms of electrode reactions [3-6]. In LSV, the potential is scanned linearly starting at the initial potential while measuring the current response. CV is an extension of LSV in that the direction of the potential scan is switched at a predetermined value and the potential is scanned again in the reverse direction to the initial value. Thus, a triangular potentialtime waveform is used in CV [4]. In this framework and according to Oldham's earlier work, there are three possible pathways for the establishment of a theoretical voltammograms [5]: analytical modelling, semi-analytical modelling and digital simulation.

For a single-step reaction and particularly for soluble-soluble systems where both oxidized and reduced species are soluble, a complementary theoretical studies by either analytical modelling, semianalytical modelling or by digital simulation, was presented and extensively discussed for reversible, quasi-reversible and irreversible processes experiments [1-10]. Randles and Ševčík were the pioneers in this field [11,12]. After that many other investigations have been carried out, especially the work of Matsuda and Ayabe for quasi-reversible systems [10,13]. A very important theoretical development of cyclic voltammetry was achieved by Nicholson and Shain in their well-known paper published in 1964 [14,15]. For soluble-insoluble system, modelling of the voltammograms was first introduced by Berzins and Delahay [16] who studied the reversible deposition on solid electrodes and derived an analytical expression for current-potential curve for single scan, using the Laplace transform and Dawson's integral. Later on, the theory was extended by Delahay to totally irreversible process [17]. Other researchers have also done similar studies but their main focus was for reversible cyclic voltammetry [18-20]. All these developments concerned the redox reactions of simple ions with a single step. However, in the case involving amalgam formation, some apparent anomalies were noted between experiment and theory for cyclic voltammetry. These facts were discussed by Beyerlein, and Nicholson [21] and others [22]. Additionally, more complicated models including ion adsorption have been proposed [23].

On the other hand, to describe the transition between fast and slow

\footnotetext{
* Corresponding authors.

E-mail addresses: affoune.abedmohamed@univ-guelma.dz (A.M. Affoune), pekka.peljo@epfl.ch (P. Peljo).
} 
electrode kinetics, the important study related to the main features in voltammetric curves such as the peak potential, the peak current and the half wave potential as a function of normalized heterogeneous rate constant was done by Matsuda and Ayabe in 1950s. Therefore, three reversibility diagrams were established for soluble-soluble systems $[10,13]$. In this direction, recently Krulic et al. presented a rough evaluation of the degree of the reversibility for one electron transfer reaction with an insoluble deposit, considering only the case when the anodic and cathodic charge transfer coefficients are both equal to 0.5 [24]. However, the effect of the combination of the rate constant and charge transfer coefficient have not been reported before this work.

Furthermore, another key aspect for kinetics study was introduced by Nicholson under the so-called working curves theory. This theory is based on correlation between the peak-to-peak potential in the cyclic voltammogram and the non-dimensional kinetic rate [15]. Thus, via the use of the Matsuda and Ayabe diagrams and Nicholson working curves, data quantifying soluble-soluble reaction kinetics could simply be determined, whatever the degree of the reversibility. Since no practical tools are available for soluble-insoluble systems, many authors [25-27] have simply resorted to use the traditional Nicholson approach in conjunction with Matsuda and Ayabe criteria to analysis experimental voltammograms under quasi-reversible condition. This approach, however, leads to misinterpretation of the experimental data.

For these reasons and to remedy these shortfalls, the purposes of the present paper are:

- Modelling of LSV responses for quasi-reversible soluble-insoluble system, employing a semi analytical method.

- Developing a general model to extract the kinetic parameters via LSV or CV data, whatever the degree of reversibility.

However, LSV models proposed in this work are only applicable to a situation where instantaneous nucleation takes place, such as metal deposition on same metal, or for example silver deposition on gold in some specific conditions [28]. If nucleation overpotential is required to induce the nucleation, followed by for example progressive $3 \mathrm{D}$ nucleation, the shape of the voltammogram will change drastically [24].

Finally, to demonstrate the accuracy and the effectiveness of our numerical model, experimental tests with the system $\mathrm{Cu}(\mathrm{I}) / \mathrm{Cu}(0)$ are performed. The accurate estimation of the electrochemical kinetics of this process is required to understand better the limitations for the nonaqueous batteries utilizing $\mathrm{Cu}(\mathrm{I}) / \mathrm{Cu}(0)$ couple as the negative redox couple $[29,30]$.

A further complication not considered in this works is the aptly named "enigma of metal deposition" [31], namely, the fact that the solvation environment of the ion changes completely upon metal deposition, while in electron transfer reactions of soluble-soluble systems only slight solvent reorganization is required. Despite this difference, some metal deposition reactions are among the fastest electrochemical reactions [31]. This problem has been described in detail by Gileadi [31-34], and has been studied both experimentally and theoretically for amalgam formation reactions, including copper deposition from various nitriles by Fawcett et al. [35,36]. To conclude, simplified Butler-Volmer type formalism employed in this work yields apparent rate constants, while more detailed analysis including corrections for double layer effects would be required to obtain the standard rate constant $[35,36]$. A solution for the "enigma of metal deposition" was recently proposed by Schmickler et al. based on theoretical calculations, proposing that univalent metal cations can approach very close to the metal surface without loss of solvation energy [37]. At this position the ion is close enough to experience a strong energetically favourable electronic interaction with the identical atoms of the electrode surface, allowing overcoming the large energy required to remove the solvation shell [37].

\section{Theory}

Prior to solving and calculating LSVs for soluble-insoluble system, we give a brief overview of Nicholson-Shain model currently used for extracting of detailed information about kinetics and mass transport for electrode reactions where both oxidized $(\mathrm{O})$ and reduced $(\mathrm{R})$ species are soluble in the solution. The reversibility factor is defined as [10]:

$\Lambda=\frac{k^{0}}{\left(\pi D_{\mathrm{O}} \frac{n \mathrm{~F}}{\mathrm{R} T} v\right)^{1 / 2}}$

For reversible reactions $(\Lambda>15)$

$I_{\mathrm{p}}=0.4463 n \mathrm{~F} A C^{*} \mathrm{o}\left(\frac{n \mathrm{~F}}{\mathrm{R} T}\right)^{1 / 2} D_{\mathrm{O}}^{1 / 2} v^{1 / 2}$

For irreversible reactions $\left(\Lambda \leq 10^{-2(1+\alpha)}\right.$, i. e. $\Lambda \leq 0.015$ if $\left.\alpha=0.5\right)$

$I_{\mathrm{p}}=0.4958 n \mathrm{~F} A C^{*} \mathrm{O}\left(\frac{\alpha n_{\alpha} \mathrm{F}}{\mathrm{R} T}\right)^{1 / 2} D_{\mathrm{O}}^{1 / 2} v^{1 / 2}$

Throughout this paper, we seek to apply Nicholson's method to generate LSV model for reactions involving an insoluble species. Nicholson's method, also called semi-analytical method, generally consists of solving the mass transport problem analytically, and deriving the theoretical voltammetric relationships numerically $[14,15]$. In the present work, our attention focused on single step electrodeposition reaction. Thus, a soluble-insoluble system of metallic ions $\mathrm{M}^{n+}$ and metal $\mathrm{M}$ couple could be represented as $\mathrm{M}^{n+}(\mathrm{sol})+n e^{-} \rightleftarrows \mathrm{M}$ (s).

Upon neglecting migration and convection contribution, the problem of the mass transport of metallic cations in Cartesian coordinates can be described by the partial differential equations (PDE):

$\frac{\partial C_{\mathrm{M}^{n+}}(x, t)}{\partial t}=D_{\mathrm{M}^{n+}} \frac{\partial^{2} C_{\mathrm{M}^{n+}}(x, t)}{\partial x^{2}}$

With the following initial and boundary conditions:

$t=0, C_{\mathrm{M}^{n+}}(x, 0)=C^{*} \mathrm{M}^{n+}$

$x \rightarrow \infty, C_{\mathrm{M}^{n+}}(\infty, t)=C^{*} \mathrm{M}^{n+}$

$x \rightarrow 0, \frac{I(t)}{n \mathrm{~F} A}=-D_{\mathrm{M}^{n+}}\left[\frac{\partial C_{\mathrm{M}^{n+}}(x, t)}{\partial x}\right]_{x=0}$

The current change is given by Butler-Volmer equation:

$$
\begin{aligned}
I(t)= & n F A k^{0}\left[C_{\mathrm{M}}(0, t) \exp \left(\frac{(1-\alpha) n F}{R T}\left(E(t)-E^{0}\right)\right)\right. \\
& \left.-C_{\mathrm{M}^{n+}}(0, t) \exp \left(\frac{-\alpha n F}{R T}\left(E(t)-E^{0}\right)\right)\right]
\end{aligned}
$$

The electrode potential $E(t)$, is swept linearly with scan rate $v$ in the negative direction starting at initial potential $E_{\mathrm{i}}$ which no electrode reactions occur, so that the potential at any time $t$ is given by:

$E(t)=E_{\mathrm{i}}-v t$

Let us consider that at the initial state, the equilibrium is achieved at the surface of electrode, so the equilibrium potential is given by the Nernst equation:

$E_{\mathrm{i}}=E_{\mathrm{eq}}=E^{0}+\frac{R T}{n F} \ln \left(\frac{a_{\mathrm{M}^{n+}}}{a_{\mathrm{M}}}\right)$

where $a_{\mathrm{M}}=1$ for pure metal and $a_{\mathrm{M}^{n+}}=\gamma_{\mathrm{M}^{n+}} \frac{C_{\mathrm{M}^{n+}}}{C^{0}}$ are respectively the activity of the metal and the corresponding cation, $\gamma_{i}$ is the activity coefficient of the species $i$ and $\mathrm{C}^{0}$ is the standard concentration of $1 \mathrm{~mol} \mathrm{~L}^{-1}$. The concentration of metal $C_{\mathrm{M}}$ in Eq. (8) can be expressed as $C_{\mathrm{M}}=a_{\mathrm{M}} C^{0}=C^{0}$ assuming that the activity coefficient of the metal is 1 .

In order to solve the above equations system (Eqs. (4)-(10)), we summarize briefly our solution procedure in three steps as follows: 
Step 1: converting Fick's differential law into Integral Equations.

Before the computation of the current-potential curve, it is necessary to know the concentration as a function of time. After applying Laplace's transform to PDE Eq. (4) under the initial condition (5) and the boundary conditions (6)-(7), the solution has the form:

$C_{\mathrm{M}^{n+}}(0, t)=C^{*}{ }^{n+}+\frac{1}{n F A \sqrt{\pi D_{\mathrm{M}^{n+}}}} \int_{0}^{t} \frac{I(\tau)}{\sqrt{t-\tau}} d \tau$

and remembering that $C_{\mathrm{M}}(0, t)=C^{0}$.

Step 2: converting the linear sweep voltammetry parameters into a non-dimensional form.

For modelling purposes, it is helpful to convert the dimensioned variables into a non-dimensional form. Thus, we set:

Dimensionless initial potential, Init:

Init $=\frac{n F}{R T}\left(E_{\mathrm{i}}-E^{0}\right)$

Dimensionless applied potential, $\Phi$ :

$\Phi=\frac{n F}{R T}\left(E(t)-E^{0}\right)=$ Init $-\sigma t$

Dimensionless scan rate, $\sigma$ :

$\sigma=\frac{n F}{R T} v$

Step 3: calculation of the current.

From combination of Eqs. (1)-(14), we obtain the general equation for the current:

$I(t)=n F A C^{*}{ }_{\mathrm{M}^{n+}}\left(\pi D_{\mathrm{M}^{n+}}\right)^{1 / 2}\left(\frac{n F v}{R T}\right)^{1 / 2} \Psi(\sigma t)$

where the dimensionless current $\Psi(\sigma t)$

$\Psi(\sigma t)=\frac{I(t)}{n F A C^{*}{ }^{n+}\left(\pi D_{\mathrm{M}^{n+}}\right)^{1 / 2}\left(\frac{n F v}{R T}\right)^{1 / 2}}$

is given by the following integral:

$\int_{0}^{\sigma t} \frac{\Psi(z)}{\sqrt{\sigma t-z}} d z=-\left[-1-\Psi(\sigma t) \frac{1}{\omega}[S(\sigma t)]^{\alpha}+[S(\sigma t)]\right]$

With the dimensionless heterogeneous rate constant defined as described in by Krulic et al. [24]:

$\omega=\frac{k^{0}}{\theta^{\alpha}\left(\pi D \frac{n F}{R T} v\right)^{1 / 2}}$

where $\theta$ is expressed as:

$\theta=\exp \left(\frac{n F}{R T}\right)\left[E_{\mathrm{i}}-E^{0}\right]=C^{*} \mathrm{M}^{n+} / C^{0}$

and

$S(\sigma t)=\exp (-\sigma t)$.

In this work the initial potential $E_{\mathrm{i}}$ is used to calculate $C^{*} \mathrm{M}^{n+}$ as activity of the metal is always taken as 1 . For performing the calculation of the integrals in the expression (17), the same numerical method developed by Nicholson and Shain [14] was used which leads to the following LSV algorithm:

$$
\begin{aligned}
& \Psi(1) \sqrt{K}+\sum_{j=1}^{K-1} \sqrt{K-j}[\Psi(j+1)-\Psi(j)] \\
& =-\frac{1}{2 \sqrt{\delta}}\left[-1-\Psi(\delta K) \omega[S(\delta K)]^{\alpha}+[S(\delta K)]^{\alpha+\beta}\right],
\end{aligned}
$$

$k=1,2,3 \ldots N$

Thus, this algorithm enables the dimensionless voltammograms to be calculated, provided that the values of Init, $\Phi, \omega$ and $\alpha$ are known.

\section{Material and methods}

\subsection{Chemicals}

All solvents and chemicals were used as received without further purification. The solvent was acetonitrile (ACN, extra dry over molecular sieves, 99.9\%, from Acros). Electrodes were made with copper wire (dia. $1 \mathrm{~mm}, \geq 99.99 \%$, from GoodFellow). The supporting electrolyte was tetraethylammonuim tetrafluoroborate $\left(\mathrm{TEABF}_{4}, 99 \%\right.$, from $\mathrm{ABCR}$ ) and the electroactive species was tetrakis(acetonitrile)copper(I) tetrafluoroborate $\left(\left[\mathrm{Cu}\left(\mathrm{CH}_{3} \mathrm{CN}\right)_{4}\right] \mathrm{BF}_{4},>98 \%\right.$, from TCI).

\subsection{Electrochemical measurements}

LSV measurements were performed by using one-compartment three-electrode cell where the reference electrode and auxiliary electrode were coils of $1 \mathrm{~mm}$ diameter copper wire and with a copper disc serving as a working electrode. The working electrode was prepared by heat-sealing $1 \mathrm{~mm}$ diameter copper wire in $2 \mathrm{~mm}$ diameter glass tube, followed by polishing with consecutively finer abrasive papers followed by different sizes of alumina, down to $0.05 \mu \mathrm{m}$ particles, on polishing cloths. The electrolyte consisted of a mixture of given concentrations of $\left[\mathrm{Cu}\left(\mathrm{CH}_{3} \mathrm{CN}\right)_{4}\right] \mathrm{BF}_{4}$ as a copper source, $100 \mathrm{mM} \mathrm{TEABF}_{4}$ as a supporting electrolyte, and acetonitrile as a solvent. All potentials are expressed vs. the $\mathrm{Cu}$ wire in the solution of the given concentration of $\left[\mathrm{Cu}\left(\mathrm{CH}_{3} \mathrm{CN}\right)_{4}\right]$ $\mathrm{BF}_{4}$. Hence, the potential of the $\mathrm{Cu}$ reference in equilibrium with $10 \mathrm{mM}$ $\mathrm{Cu}^{+}$solution in acetonitrile on the "non-aqueous standard copper electrode" scale (SCuE), i.e. vs. $\mathrm{Cu}^{+}$solution with the activity of 1 in equilibrium with $\mathrm{Cu}$ in acetonitrile is assumed to follow the Nernst equation considering the $\gamma_{\mathrm{Cu}^{+}}=1$, i.e. the potential of the reference is $-0.118 \mathrm{~V}$ vs. SCuE in acetonitrile. To establish the relation with the ferrocene $(\mathrm{Fc})$ scale recommended by IUPAC, the potential of the $\mathrm{Fc}^{+}$/ Fc couple vs. $\mathrm{Cu} / 10 \mathrm{mM} \mathrm{Cu}^{+}$in ACN was measured as $0.69 \pm 0.01 \mathrm{~V}$, i.e. the potential of our reference electrode is $-0.69 \mathrm{~V} \mathrm{vs.} \mathrm{Fc}^{+} / \mathrm{Fc}$. LSV data for the reduction of copper (I) in acetonitrile were recorded with an Autolab model PGSTAT302N potentiostat equipped with the SCAN250 analog scan generator module. We point out that, all experimental steps (solution preparation, LSV measurement) were performed at ambient temperature $\left(25^{\circ} \mathrm{C}\right)$ under anaerobic conditions using a nitrogen filled glove box, utilizing positive feedback $i R$ compensation.

\section{Results and discussion}

\subsection{Theoretical results}

\subsubsection{Effect of the kinetic rate}

Generally, the critical parameters used for the diagnosis of electron transfer reactions via linear sweep voltammograms are the magnitudes of the peak current, the peak potential and the half peak width. These LSV responses may depend on multiple variables including the charge transfer coefficient $\alpha$, the potential sweep rate $v$, and the heterogeneous standard rate constant $k^{0}$. In the present paper, the mutual influence of $k^{0}$ and $v$ is expressed through the magnitude of the dimensionless parameter, $\omega$, defined by Eq. (18). The effects of varying $\omega$ for a constant value of $\alpha$ on the position, high and width of peak are examined and shown in Fig. 1, in which three distinct regions can be seen:

1) $\omega \geq 10^{3}$

For which, the dimensionless current-potential curves become insensitive to the dimensionless rate constant (curves a, b, c in the inset of Fig. 1). The dimensionless peak current, $\pi^{1 / 2} \Psi_{\mathrm{p}}$, takes a constant value of -0.6105 , as reported by Berzin and Delahay [16] for reversible system. 


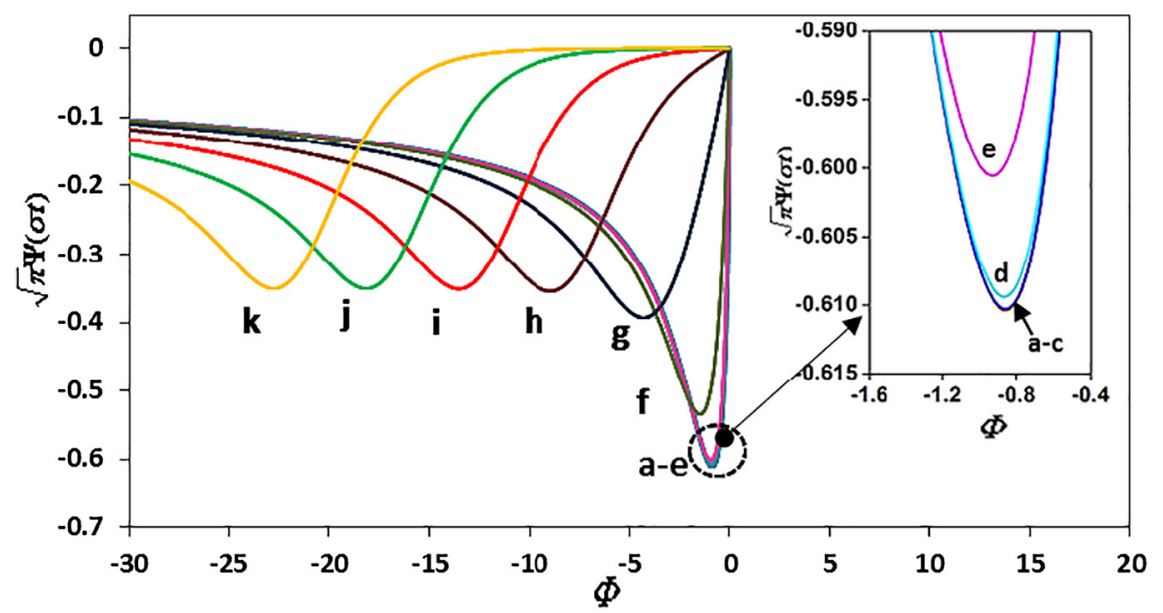

Fig. 1. Calculated linear sweep voltammograms for various value of $\omega$ with $\alpha=0.5$; a: $\omega=10^{5}$, b: $\omega=10^{4}$, c: $\omega=10^{3}$, d: $\omega=10^{2}$, e: $\omega=10^{1}$, f: $\omega=1$, g: $\omega=10^{-1}, \mathbf{h}: \omega=10^{-2}, \mathbf{i}: \omega=10^{-3}, \mathbf{j}: \omega=10^{-4}, \mathbf{k}: \omega=10^{-5}$.

2) $\omega \leq 10^{-3}$

In this region, the calculated linear sweep voltammograms retain their shape (curves i-k in Fig. 1). The peak half width, $\Delta \Phi_{\mathrm{p} / 2}$, remains unchanged and the dimensionless peak current $\left(\pi^{1 / 2} \Psi_{\mathrm{p}}\right)$ keeps stable in value of -0.350 , which is in good agreement with literature values for irreversible systems [17]. Contrary to the peak shape, the peak position is shifted towards more negative potentials as $\omega$ decreases.

3) $10^{-3}<\omega<10^{3}$

Within these two kinetic regions (curves $\mathrm{d}-\mathrm{h}$ in Fig. 1), a decrease in $\omega$ leads to a diminution in the peak height, an increase in peak half width, and a shift of the dimensionless peak potential $\left(\Phi_{\mathrm{p}}\right)$ towards more negative values. These observations refer to characteristics of quasi-reversible waves.

\subsubsection{Effect of the charge transfer coefficient}

A series of theoretical voltammograms in which the charge transfer coefficient $\alpha$ is varied, are displayed in Fig. 2. Note that the impact of $\alpha$ on the peak parameters depends upon the magnitude of the dimensionless kinetic rate $\omega$.

- For $\omega=10^{3}$, no change occurs in current and potential values with $\alpha$ varying from 0.2 to 0.8 . This observation agrees reasonably with previous studies for reversible system [16].

- For $\omega=1$, an increase in $\alpha$ value from 0.2 to 0.8 is followed by a slightly increase in the dimensionless peak current (in absolute value), while the peak potential remains almost constant.

- For $\omega=10^{-3}$, the effect of the electron transfer coefficient has an appreciable influence on the height, position, and the shape of the peak.

4.1.3. Kinetics curves: coupling effects of kinetic rate and charge transfer coefficient

It is clear from the above results that the voltammograms in the intermediate region, $10^{-3}<\omega<10^{3}$, are qualitatively different. Furthermore, until now, as no characteristic equations or practical tools for analysis of experimental voltammograms for quasi-reversible systems has been reported, our goal in this section is to provide a general, simple and direct model capable to solve the problem of the determination of the kinetic parameters in the case of quasi-reversible solubleinsoluble systems. The same issue was encountered before for solublesoluble system, and the solution was offered by suggesting series of working curves which covered the range from reversible to irreversible
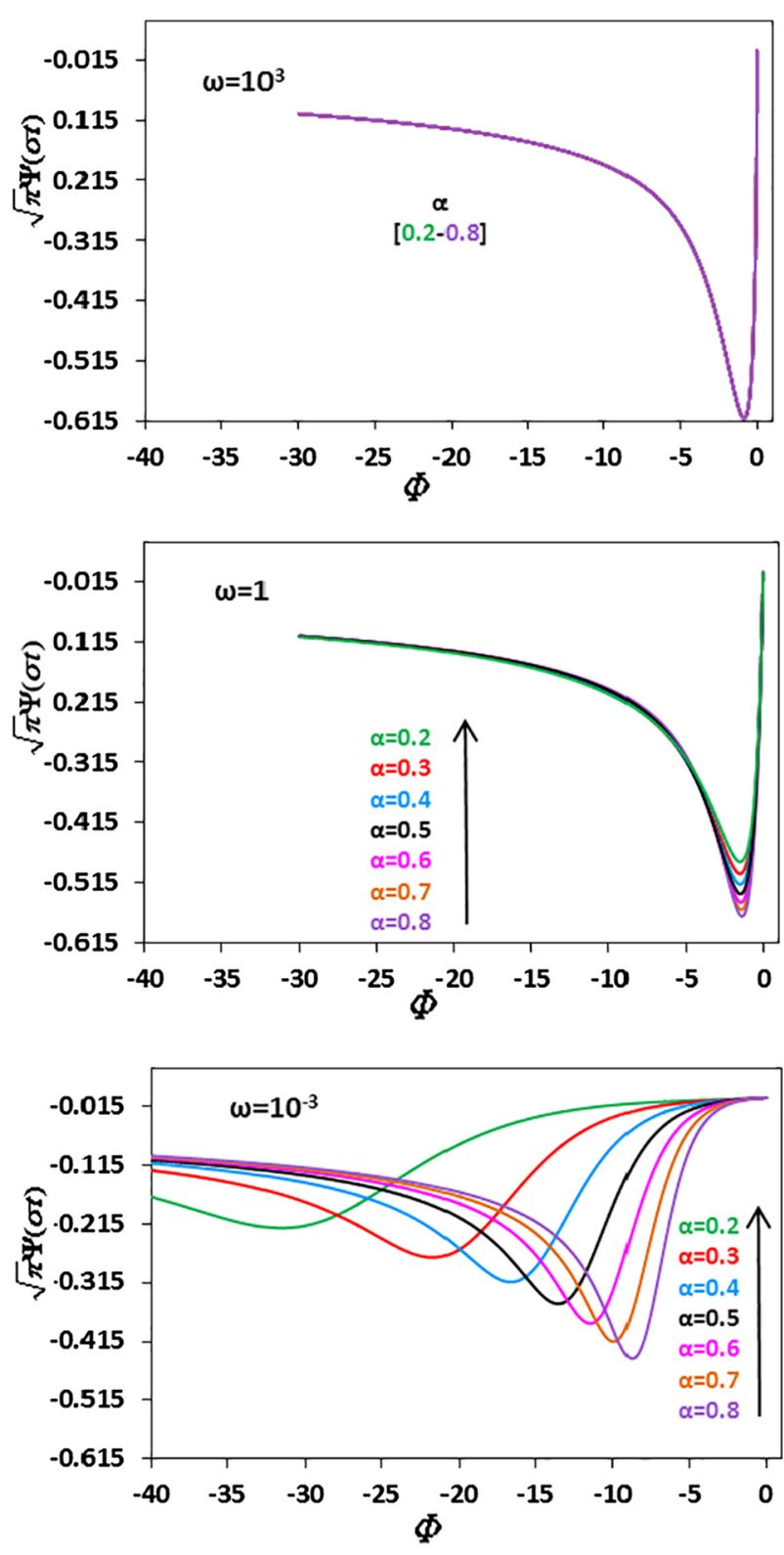

Fig. 2. The effect of transfer coefficient $\alpha$ on theoretical voltammograms. 


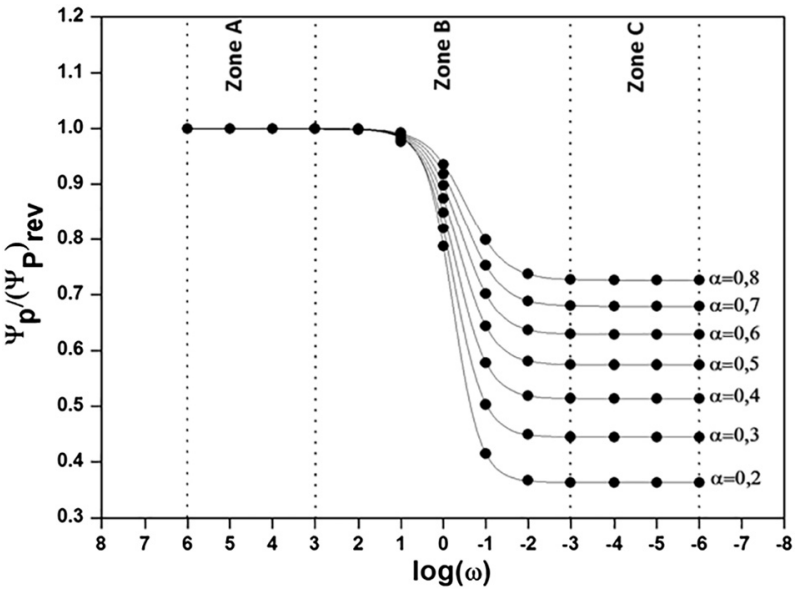

Fig. 3. Variation of the peak current ratio, $\Psi_{\mathrm{p}} /\left(\Psi_{\mathrm{p}}\right)_{\mathrm{rev}}$, as the function of the dimensionless rate constant for several values of $\alpha$. Solid lines are best fits to the sigmoidal Boltzmann functions with a correlation coefficient of 0.99. Zones A, $\mathrm{B}, \mathrm{C}$ denote the reversible, quasi-reversible and totally irreversible zones, respectively.

behaviour.

Likewise, in the next set of figures, we have constructed three kinetic curves for single electron transfer process, in which we show quantitatively the coupling effects of the dimensionless kinetic rate $(\omega)$ and the electron transfer coefficient $\alpha$ on LSV responses. The values of $\omega$ in these calculations was varied from $10^{6}$ to $10^{-6}$ while the values of $\alpha$ was varied from 0.2 to 0.8 . Fig. 3 shows the plot of the peak current ratio $\Psi_{\mathrm{p}} /\left(\Psi_{\mathrm{p}}\right)_{\text {rev }}$ vs. $\log (\omega)$, where $\left(\Psi_{\mathrm{p}}\right)_{\text {rev }}$ is the reversible dimensionless peak current. Fig. 4 shows quantitatively the variation of the peak shape through the half peak width changes, $\Delta \Phi_{\mathrm{p} / 2}=\frac{n \mathrm{~F}}{\mathrm{R} T}\left(E_{\mathrm{p}}-E_{\mathrm{p} / 2}\right)$ as a function of $\log (\omega)$ and $\alpha$ and Fig. 5 describes how the cathodic peak position $\eta_{\mathrm{p}}, \eta_{\mathrm{p}}=\frac{n \mathrm{~F}}{\mathrm{R} T}\left(E_{p}-E_{e q}\right)$, changes as a function of $\log (\omega)$ and $\alpha$.

It is apparent from Fig. 4, that the plots of the LSV responses as a function of both, the dimensionless heterogeneous rate constant $\omega$ and the charge transfer coefficient $\alpha$, exhibit sigmoidal shapes. Data were then fitted perfectly by the sigmoidal Boltzmann functions:

$\frac{\Psi_{\mathrm{p}}}{\left(\Psi_{\mathrm{p}}\right)_{\mathrm{rev}}}=1+\frac{\left(0.811 \alpha^{0.5}-1\right)}{1+\exp \left[\frac{X-(-0.528 \alpha-0.099)}{0.477 \alpha^{0.248}}\right]}$

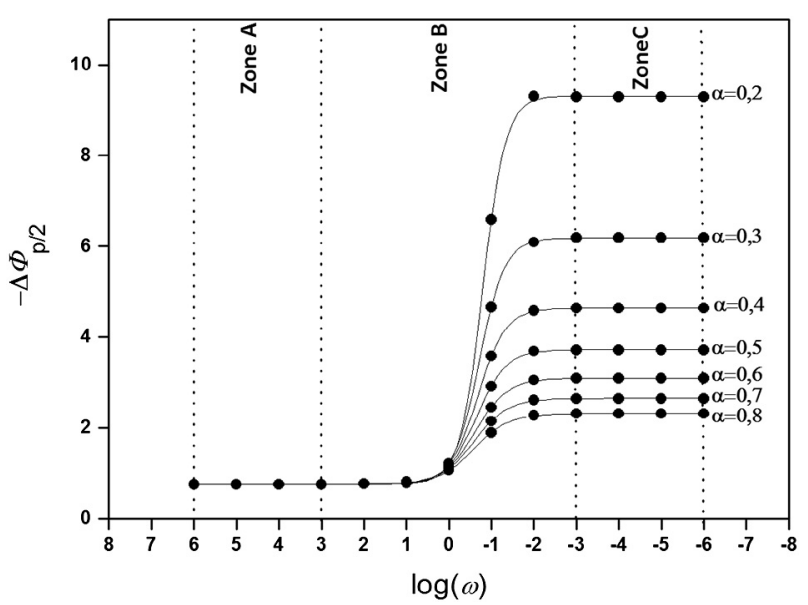

Fig. 4. Dependence of the half peak width of linear sweep voltammograms $\left(\Delta \Phi_{\mathrm{p} / 2}\right)$ on the logarithm of the kinetic parameter $\omega$ for various $\alpha$ values. Solid lines are best fits to the sigmoidal Boltzmann functions with a correlation coefficient of 0.98 .

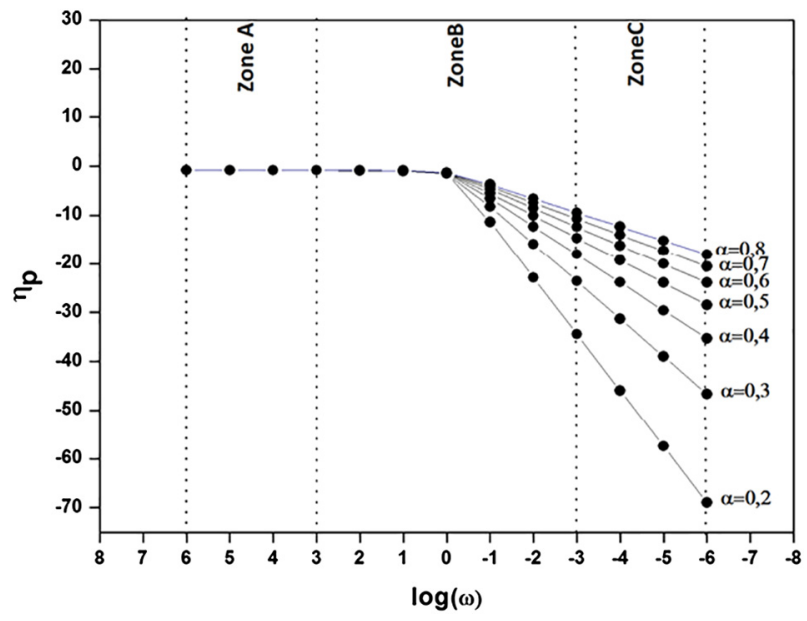

Fig. 5. Plots of the reduction peak position $\left(\eta_{\mathrm{p}}\right)$ against $\log (\omega)$ for various $\alpha$ values.

$-\Delta \Phi_{\mathrm{p} / 2}=0.770+\frac{\left(1.857 \alpha^{-1}-0.770\right)}{1+\exp \left[\frac{X+\left(0.557 \alpha^{-0.216}\right)}{0.445 \alpha^{0.316}}\right]}$

where: $X=\log (\omega)$. Each of these fit models enables voltammetric quantification of the electrode kinetics from simple peak current and peak potential measurements of the experimental linear sweep voltammograms for either reversible, quasi reversible or totally irreversible electron transfer process, provided that the value of $\alpha$ is known or can be estimated accurately. Also, from Figs. 3-5, the following kinetic zones properties can be concluded:

In the zone A both the current and the peak potentials are independent of the values of $\alpha$ and the dimensionless kinetic rate $\omega$. All peak parameters $\eta_{\mathrm{p}}, \Psi_{\mathrm{p}}$, and $\Delta \Phi_{\mathrm{p} / 2}$, reach their reversible values, yielding to the following reversible criteria:

For $\omega \geq 10^{3}$

$I_{\mathrm{p}}=0.6105 n F A C^{*} \mathrm{M}^{n+}\left(D_{\mathrm{M}^{n+}}\right)^{1 / 2}\left(\frac{n F v}{R T}\right)^{1 / 2}$

$E_{\mathrm{p}}=E_{\mathrm{eq}}-0.854 \frac{R T}{n F}$

$E_{\mathrm{p}}-E_{\mathrm{p} / 2}=-0.77 \frac{R T}{n F}$

These criteria for kinetically reversible system are in excellent agreement with those of previous theoretical investigations [16,18-20,38].

Quasi-reversible features are observed in the zone B $(-3<\log$ $\omega<3$ ), where marked changes in the various peak parameters as a function of both $\log \omega$ and $\alpha$ are evident. As shown in Figs. 3-4, the peak height ratio increases while the half peak width decreases for increasing values of $\alpha$. Both values decrease with decreasing values of $\log \omega$. This effect of the charge transfer coefficient can be interpreted by the change in the symmetry of the energy barrier.

Irreversible behaviour is evident in the zone $\mathrm{C}$, where the peak current and half peak width remain constant with decreasing $\log \omega$ for specific values of $\alpha$ while the peak potential continues to decrease linearly as the function of decreasing $\log \omega$. As for the quasi-reversible case, the peak height ratio and peak potential increase and the half peak width decreases for increasing values of $\alpha$. Therefore, the following conclusions can be drawn:

For $\omega \leq 10^{-3}$ :

$\frac{\Psi_{\mathrm{p}}}{\left(\Psi_{\mathrm{p}}\right)_{\mathrm{rev}}}=0.811 \alpha^{1 / 2}$ 
$\Delta \Phi_{\mathrm{p} / 2}=1.857 \alpha^{-1}$

These equations show a very good agreement with those obtained by Delahay for irreversible soluble-insoluble redox system [17].

A further key point to consider in Fig. 5, which was also observed and demonstrated by Krulic et al. [24], is that in the region when log $\omega \leq-1$ ), the magnitude of $\eta_{\mathrm{p}}$ depends linearly on the logarithm of the kinetic parameter $\omega$. Therefore, by using linear regression approximations, the dependency of $\eta_{\mathrm{p}}$ on $\omega$ and $\alpha$ could be expressed as follows:

$\alpha \eta_{p}=2.303 X-[0.115 \log (\alpha)+0.78]$

This equation coincides with that established by Krulic for $\alpha=0.5$ [24], it can be used for estimation of the kinetic rate constant, in particularly for quasi-reversible and irreversible electrodeposition processes if the value of $\alpha$ is known or can be estimated.

\subsection{Experiment-theory comparison}

In order to test the validity of our numerical approach to extract kinetic and mass transport parameters from LSV data, an example of $\mathrm{Cu}$ electrodeposition reaction in organic solution is presented. The $\mathrm{Cu}$ system includes a simple one-electron transfer reaction according to [29]:

$\mathrm{Cu}^{+}+\mathrm{e}^{-} \rightarrow \mathrm{Cu}(0)$ although, the removal of the complexing solvent should also be considered [35,36]. Fig. 6a depicts typical LSV profiles collected at different scan rates for reduction reaction, $\mathrm{Cu}(\mathrm{I}) / \mathrm{Cu}(0)$, in acetonitrile. Regarding to the peak heights, an increasing trend was observed on increasing the scan rates. Furthermore, the peak potential was seen to shift gradually towards more negative potential values over $25-200 \mathrm{mV} / \mathrm{s}$ scan rate suggesting a quasi-reversible character. To deduce the mass-transport and kinetic proprieties of the $\mathrm{Cu}(\mathrm{I}) / \mathrm{Cu}(0)$ system, the unknown parameters values: $D_{\mathrm{Cu}(\mathrm{I})}, \alpha$ and $k^{0}$ need to be derived. There are two ways for the calculation of $D_{\mathrm{Cu}(\mathrm{I})}, \alpha$ and $k^{0}$ : by either computationally or by adjusting curves based only on LSV algorithm (Eq. (21)) and different combinations of $D_{\mathrm{Cu}(\mathrm{I})}, \alpha$ and $k^{0}$.

\subsubsection{Utilization of working curves}

First, we used the common employed procedure, the semi-integrative voltammetry, for the calculation of $\mathrm{Cu}(\mathrm{I})$ diffusion coefficient and a direct Tafel analysis for the measurement of the transfer coefficient $\alpha$. Fig. 6b shows a sigmoidal curve (dotted line) obtained from semi-integration of typical voltammetric current recorded at $100 \mathrm{mV} / \mathrm{s}$. It should be noted that we have employed the Saila methodology [39] for establishing semi-integrated plots. As shown in Fig. 6b and as per principle of semi-integration technique, the semi-integration of the voltammetric current responses with respect to time yields to the sigmoidal-type curve with a plateau. This plateau level represents the limiting semi-integral current, of height $\mathrm{m}^{*}$ :
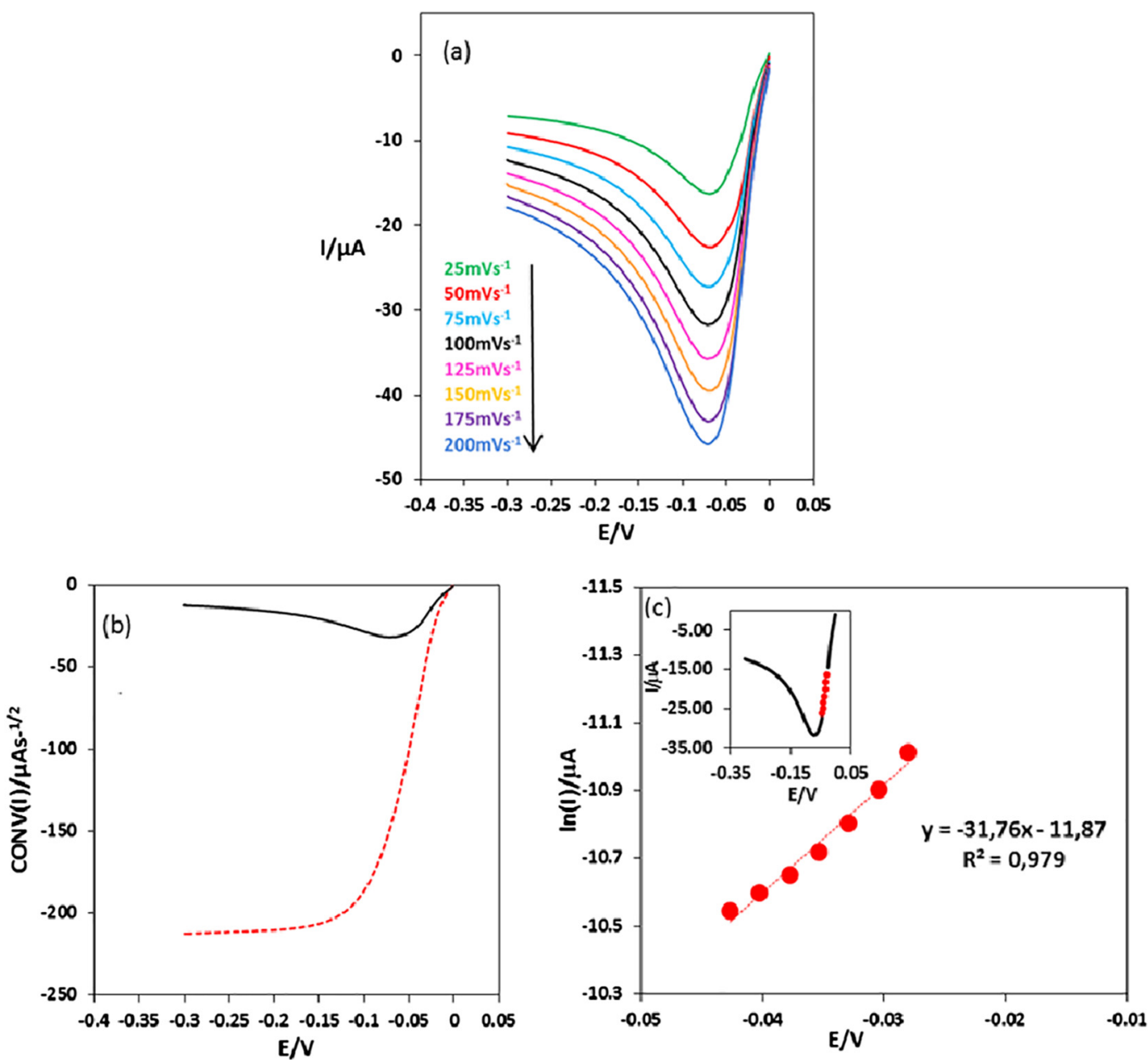

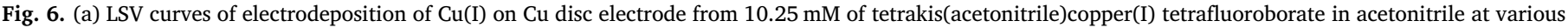

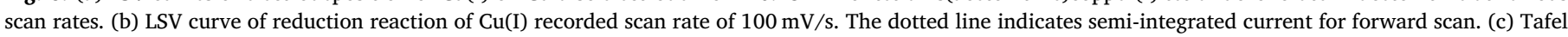

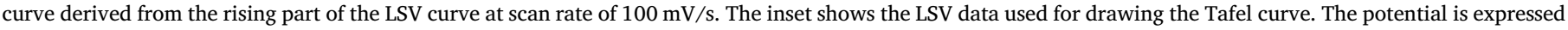
vs. the $\mathrm{Cu}$ reference in equilibrium with $10.25 \mathrm{mM} \mathrm{Cu}^{+}$in solution, i.e. $-0.118 \mathrm{~V}$ vs. standard copper electrode in acetonitrile or $-0.69 \mathrm{~V}$ vs. Fc ${ }^{+} / \mathrm{Fc}$. 
Table 1

Kinetic-mass transport parameters for $\mathrm{Cu}(\mathrm{I}) / \mathrm{Cu}(0)$ redox couple.

\begin{tabular}{|c|c|c|c|c|c|c|c|c|}
\hline \multirow[t]{4}{*}{$\mathrm{Cu}(\mathrm{I}) / \mathrm{Cu}(0)$ system } & \multicolumn{2}{|c|}{$D_{\mathrm{Cu}(\mathrm{I})}\left[10^{-9} \mathrm{~m}^{2} \mathrm{~s}^{-1}\right]$} & \multicolumn{2}{|l|}{$\alpha$} & \multicolumn{4}{|c|}{$k^{0}\left[10^{-5} \mathrm{~cm} \mathrm{~s}^{-1}\right]$} \\
\hline & SI & Fit & Tafel & Fit & Working & & & Fit \\
\hline & & & & & Eq. (22) & Eq. (23) & Eq. (29) & \\
\hline & 1.75 & 1.70 & 0.82 & 0.80 & 3.84 & 4.99 & 7.73 & 5.12 \\
\hline
\end{tabular}

$m^{*}=n F A C_{\mathrm{M}^{n+}}^{*} D_{\mathrm{M}^{n+}}^{1 / 2}$

and the diffusion coefficient of $\mathrm{Cu}(\mathrm{I})$ in acetonitrile at $25^{\circ} \mathrm{C}$ was computed utilizing this equation (as shown in Table 1) by considering the following experimental values: $n=1, C_{\mathrm{Cu}^{+}}{ }^{*}=10.25 \mathrm{mM}$. Fig. $6 \mathrm{c}$ depicts the representation of the Tafel plot obtained from experimental LSV data, at a scan rate of $100 \mathrm{mV} / \mathrm{s}$. Using the slope calculated from Fig. $6 \mathrm{c}$ and the Tafel equation, the value of $\alpha$ was calculated and the result is reported in Table 1 . In the next step, a more detailed examination of the voltammetric peaks allowed us to quantify the standard rate constant $\left(k^{0}\right.$ in $\left.\mathrm{cm}^{2} / \mathrm{s}\right)$ for the $\mathrm{Cu}(\mathrm{I}) / \mathrm{Cu}(0)$ redox couple according to the models presented in Section 4.1.3. The results are listed in Table 1. With these values, Eqs. (22), (23) and (29) were used to obtain the final value for the $k^{0}$.

\subsubsection{Fit and simulation}

The parameters, $D_{\mathrm{Cu}(\mathrm{I})}, \alpha$ and $k^{0}$ were also obtained by comparison of theoretical and the experimental linear scan voltammograms (in $100 \mathrm{mM} \mathrm{TEABF}_{4}$ and $\left.10 \mathrm{mM} \mathrm{Cu}\left(\mathrm{CH}_{3} \mathrm{CN}\right)_{4} \mathrm{BF}_{4}\right)$, using the LSV model algorithm with a series of adjustments in the input values. For this purpose, theoretical voltammograms were obtained by utilizing the diffusion coefficient of $\mathrm{Cu}$ ions within the range reported in the literature [40], and varying the values for $k^{0}$ and $\alpha$ for the $\mathrm{Cu}(\mathrm{I}) / \mathrm{Cu}(0)$ system as these values have not been reported previously in the literature. In our work, $\alpha$ was varied from 0.1 to 0.9 while the dimensionless kinetic rate was varied from $10^{-3}$ to $10^{3}$.

In the purpose to compare the two approaches: i) theoretical fitting and ii) working curves, and to illustrate the LSVs are obtained with the parameters from Eqs. (22), (23) and (29), an analysis study was performed first with the voltammetric data collected at $100 \mathrm{mV} / \mathrm{s}$. To achieve best fit to experimental LSV data obtained at $100 \mathrm{mV} / \mathrm{s}$, we tested various values of $D_{\mathrm{Cu}(\mathrm{I})}, \alpha$ and $k^{0}$. With the following parameters: $D_{\mathrm{Cu}(\mathrm{I})}=1.7 \times 10^{-9} \mathrm{~m}^{2} \mathrm{~s}^{-1}, \alpha=0.8$ and $k^{0}=5.12 \times 10^{-5} \mathrm{~cm} \mathrm{~s}^{-1}$ the resultant theoretical voltammogram is in very good agreement with the experimental LSV curve (see Fig. 7a). To demonstrate the applicability of the working curves, theoretical voltammograms were calculated with Eq. (21) with the parameters shown in Table 1 and the results are presented in Fig. 7b. Better agreement was obtained by using Eqs. (22) and (23) than by Eq. (29). The small discrepancy in kinetic prediction using Eq. (29) could be attributed to the dimensionless kinetic rate constant $\omega$ of copper reduction which was just out of range of

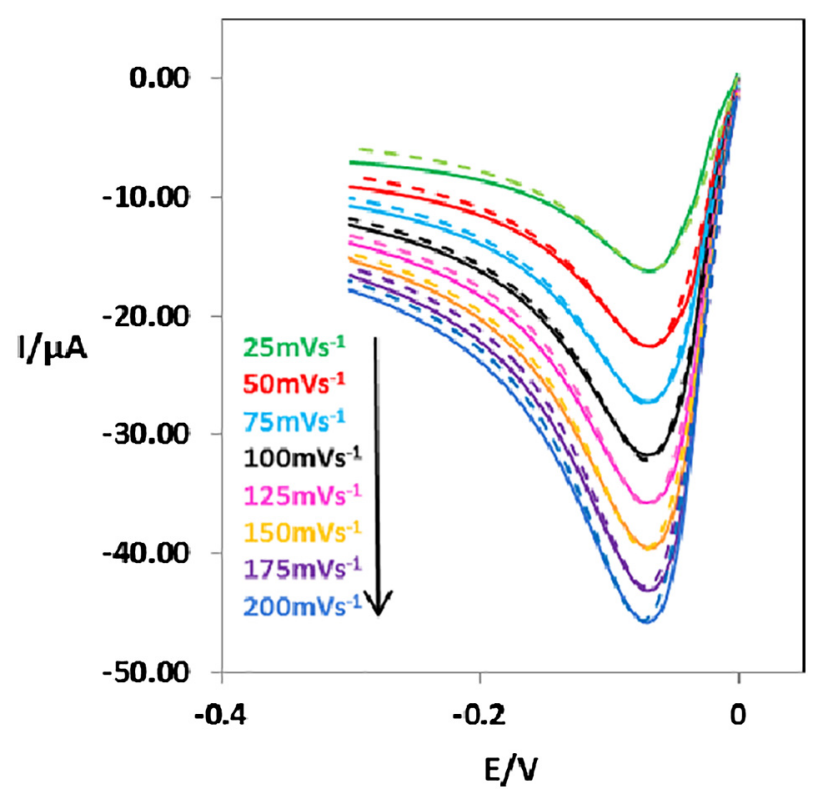

Fig. 8. Fits obtained between experimental LSV data (solid line) and theoretical LSV data (dashed line) generating using Eq. (21), for parameters ( $\alpha$ and $D_{\mathrm{Cu}(\mathrm{I})}$ ) shown in Table 1 , under different scan rates.

applicability of Eq. (29). We have found above that Eq. (29) is valid for $\omega \leq 0.1$; however the $\omega$ value calculated for Cu reaction is 0.15 .

Table 1 shows the obtained parameters for the reduction of $\mathrm{Cu}(\mathrm{I})$ to $\mathrm{Cu}(0)$ in acetonitrile at $100 \mathrm{mVs}^{-1}$. The obtained $D_{\mathrm{Cu}(\mathrm{I})}$ values are similar with the values previously reported in literature [40], although slightly higher diffusion coefficient was obtained from semi-integration analysis. Furthermore, the calculated values of $k^{0}$ indicated that the $\mathrm{Cu}$ deposition process in acetonitrile is quasi-reversible.

Likewise, the kinetics of the $\mathrm{Cu}(\mathrm{I}) / \mathrm{Cu}(0)$ system were analyzed at all scan rates. The use of Eqs. (22), (23) and (29), yielding average values of the standard rate constant $k^{0}: 4.86( \pm 0.68) \times 10^{-5}$, $4.63( \pm 0.22) \times 10^{-5}$ and $7.76( \pm 0.76) \times 10^{-5} \mathrm{~cm} / \mathrm{s}$, respectively. Excellent fits were reproduced between the experimental LSVs and simulated ones under various scan rates (see Fig. 8) and the average fitted $k^{0}$ value was found: $5.43( \pm 0.52) \times 10^{-5} \mathrm{~cm} / \mathrm{s}$. These results
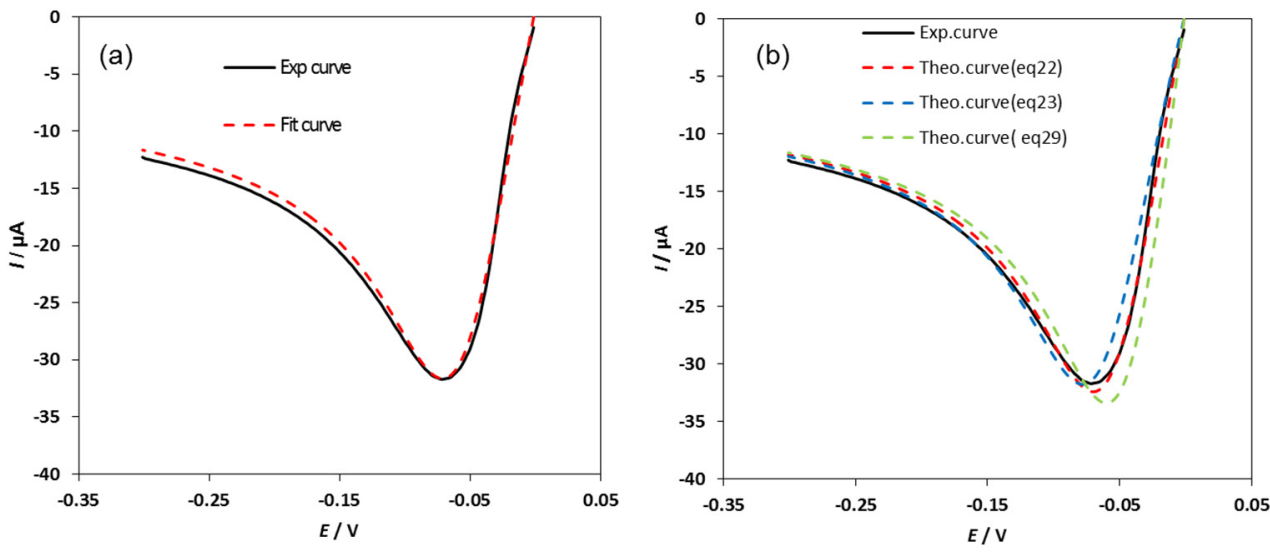

Fig. 7. (a) A comparisons between theoretical linear scan voltammogram and experimental voltammogram obtained and recorded at $100 \mathrm{mV} / \mathrm{s}$ scan rate. The parameters used for theoretical prediction are: $D_{\mathrm{Cu}(\mathrm{I})}=$ $1.70 \times 10^{-9} \mathrm{~m}^{2} \mathrm{~s}^{-1}, \quad \alpha=0.8 \quad$ and $k^{0}=5.12 \times 10^{-5} \mathrm{~cm} \mathrm{~s}^{-1}$. (b) Comparison of theoretical LSV responses with experimental data using Fit models presented by Eqs. (22), (23) and (29). Parameters used for LSV modelling: $D_{\mathrm{Cu}(\mathrm{D})}=1.75 \times 10^{-9} \mathrm{~m}^{2} \mathrm{~s}^{-1} \alpha=0.82$ and $k^{0}=3.83 \times 10^{-5} \mathrm{~cm} \mathrm{~s}^{-1}$ calculated from Eq. (22), $\quad k^{0}=3.83 \times 10^{-5} \mathrm{~cm} \mathrm{~s}^{-1}$ calculated from Eq. (23) and $k^{0}=5.88 \times 10^{-5} \mathrm{~cm} \mathrm{~s}^{-1}$ calculated from Eq. (29). The potential is expressed vs. the $\mathrm{Cu}$ reference in equilibrium with $10.25 \mathrm{mM} \mathrm{Cu}^{+}$in solution, i.e. $-0.118 \mathrm{~V}$ vs. standard copper electrode in acetonitrile. 
confirm the above conclusions, and reveal that the working curves provided in this work can be used to accurately analyze the linear scan voltammetric current responses for quasi-reversible soluble/insoluble system.

The high transfer coefficient value of 0.8 obtained in this work is in line of the values obtained for $\mathrm{Cu}$ deposition on amalgams from nitrile solvents, indicating that the reaction site is located in the inner part of the electric double layer $[35,36]$. However, the apparent rate constants measured in this work for $\mathrm{Cu}$ deposition are three orders of magnitude smaller than the standard rate constants obtained for copper deposition on mercury $[35,36]$, indicating that ion transfer into mercury is easier than nucleation on copper surface.

\section{Conclusion}

In summary, using an extension of Nicholson's method, a new LSV algorithm has been proposed for use in the computing of voltammograms in the case of soluble-insoluble system where the electron transfer takes place in a single step. The effect of the kinetic parameters on the LSV responses has been examined. Through the variation of the peak parameters with dimensionless kinetic rate $(\omega)$ and the transfer coefficient $(\alpha)$, series of kinetic curves have been established. The obtained results showed that according to the magnitude of the dimensionless rate constant the various LSV responses limitation could be divided into three zones:

Zone A: $\omega \geq 10^{3}$, reversible process.

Zone B: $10^{-3}<\omega<10^{3}$, quasi-reversible process.

Zone C: $\omega \leq 10^{-3}$, irreversible process.

Moreover, we offer here three working curves to extract kinetic details with high accuracy and simplicity to use, as only the experimental values corresponding to the peak high, peak position and peak width are required.

LSV models proposed in this work are applicable only to a situation where instantaneous nucleation takes place, such as metal deposition on same metal, or for example silver deposition on gold in some specific conditions [28]. If nucleation overpotential is required to induce the nucleation, followed by for example progressive 3D nucleation, the shape of the voltammogram will change drastically, and the described kinetic curves and LSV models are no longer valid [24]. On the other hand, we point out that although this paper was limited to investigation of the soluble-insoluble system during a linear scan, the LSV algorithm could be extend with some modifications to model also CV, but in this case the activity of the metal as the function of surface coverage would be required.

\section{Acknowledgements}

I. A. is grateful for the financial support from Ministère de l'Enseignement Supérieur et de la Recherche Scientifique, République Algérienne Démocratique et Populaire, Programme National Exceptionnel, Algeria. P.P. and S.M. acknowledge the financial support from the Swiss National Science Foundation under Grant Ambizione Energy 160553.

\section{Appendix 1. Nomenclature}

\section{Notations}

$\begin{array}{ll}C_{\mathrm{M}} & \text { Concentration of the metal M } \\ C_{\mathrm{M}^{n+}} & \text { Concentration of the metallic ions } \mathrm{M}^{\mathrm{n}+} \\ C^{*} \mathrm{M}^{n+} & \text { Bulk concentration of the metallic ions } \mathrm{M}^{\mathrm{n}+} \\ C^{0} & \text { Standard concentration } \\ D_{\mathrm{M}^{n+}} & \text { Diffusion coefficient of metallic ions } \mathrm{M}^{\mathrm{n}+} \\ A & \text { Electroactive surface area } \\ n & \text { Number of electrons } \\ F & \text { Faraday's constant }\end{array}$

$R \quad$ Universal gas constant

$T \quad$ Absolute temperature

$k^{0} \quad$ Standard rate constant

I Current

$E(t) \quad$ Electrode potential

$E_{\mathrm{i}} \quad$ Initial potential

$E^{0} \quad$ Standard potential

$E_{\text {eq }} \quad$ Equilibrium potential

$v \quad$ Potential scan rate

$t \quad$ Time

\section{Greek letters}

$\alpha \quad$ Charge transfer coefficient

1- $\alpha \quad$ Anodic charge transfer coefficient

$\omega \quad$ Dimensionless kinetic rate parameter

$\Psi \quad$ Dimensionless current

$\eta \quad$ Dimensionless overvoltage $\left(\eta=n F / R T\left(E-E_{\mathrm{eq}}\right)\right)$

$\sigma \quad$ Dimensionless scan rate

$\gamma_{\mathrm{M}^{n+}} \quad$ Activity coefficient of the metallic ions $\mathrm{M}^{\mathrm{n}+}$

$\delta_{k} \quad$ Small time interval

\section{References}

[1] L.K. Bieniasz, Analysis of the applicability of the integral equation method in the theory of transient electroanalytical experiments for homogeneous reaction-diffusion systems: the case of planar electrodes, J. Electroanal. Chem. 657 (2011) 91-97, http://dx.doi.org/10.1016/J.JELECHEM.2011.03.027.

[2] L.K. Bieniasz, Use of dynamically adaptive grid techniques for the solution of electrochemical kinetic equations: part 5. A finite-difference, adaptive space/time grid strategy based on a patch-type local uniform spatial grid refinement, for kinetic models in one-dimensional space geometry, J. Electroanal. Chem. 481 (2000) 115-133, http://dx.doi.org/10.1016/S0022-0728(99)00460-X.

[3] D. Yan, M.Z. Bazant, P.M. Biesheuvel, M.C. Pugh, F.P. Dawson, Theory of linear sweep voltammetry with diffuse charge: unsupported electrolytes, thin films, and leaky membranes, Phys. Rev. E 95 (2017) 33303, http://dx.doi.org/10.1103/ PhysRevE.95.033303.

[4] D.J. Gavaghan, A.M. Bond, A complete numerical simulation of the techniques of alternating current linear sweep and cyclic voltammetry: analysis of a reversible process by conventional and fast Fourier transform methods, J. Electroanal. Chem. 480 (2000) 133-149, http://dx.doi.org/10.1016/S0022-0728(99)00476-3.

[5] K.B. Oldham, J.C. Myland, Modelling cyclic voltammetry without digital simulation, Electrochim. Acta 56 (2011) 10612-10625, http://dx.doi.org/10.1016/J. ELECTACTA.2011.05.044.

[6] A. Samin, E. Lahti, J. Zhang, Analytical solutions of the planar cyclic voltammetry process for two soluble species with equal diffusivities and fast electron transfer using the method of eigenfunction expansions, AIP Adv. 5 (2015) 87141, http://dx. doi.org/10.1063/1.4928862.

[7] A.M. Keightley, J.C. Myland, K.B. Oldham, P.G. Symons, Reversible cyclic voltammetry in the presence of product, J. Electroanal. Chem. 322 (1992) 25-54, http://dx.doi.org/10.1016/0022-0728(92)80065-C.

[8] P.J. Mahon, J.C. Myland, K.B. Oldham, A fresh approach to voltammetric modelling, J. Electroanal. Chem. 537 (2002) 1-5, http://dx.doi.org/10.1016/S00220728(02)01263-9.

[9] A. Eswari, L. Rajendran, Mathematical modeling of cyclic voltammetry for EC reaction, Russ. J. Electrochem. 47 (2011) 181-190, http://dx.doi.org/10.1134/ S1023193511020078.

[10] A.J. Bard, L.R. Faulkner, Electrochemical Methods: Fundamentals and Applications, 2nd ed., John Wiley \& Sons, New York, 2001.

[11] A. Ševčík, Oscillographic polarography with periodical triangular voltage, Collect. Czechoslov. Chem. Commun. 13 (1948) 349-377, http://dx.doi.org/10.1135/ cccc19480349.

[12] J.E.B. Randles, A cathode ray polarograph. Part II.-the current-voltage curves, Trans. Faraday Soc. 44 (1948) 327-338, http://dx.doi.org/10.1039/ TF9484400327.

[13] H. Matsuda, Y. Ayabe, Zur Theorie der Randles-Sevčikschen KathodenstrahlPolarographie, Zeitschrift Für Elektrochemie, Berichte Der Bunsengesellschaft Für Phys. Chemie. 59 (1955) 494-503, http://dx.doi.org/10.1002/BBPC. 19550590605.

[14] R.S. Nicholson, I. Shain, Theory of stationary electrode polarography. Single scan and cyclic methods applied to reversible, irreversible, and kinetic systems, Anal. Chem. 36 (1964) 706-723, http://dx.doi.org/10.1021/ac60210a007.

[15] R.S. Nicholson, Theory and application of cyclic voltammetry for measurement of electrode reaction kinetics, Anal. Chem. 37 (1965) 1351-1355, http://dx.doi.org/ 10.1021/ac60230a016.

[16] T. Berzins, P. Delahay, Oscillographic polarographic waves for the reversible deposition of metals on solid electrodes, J. Am. Chem. Soc. 75 (1953) 555-559, http://dx.doi.org/10.1021/ja01099a013. 
[17] P. Delahay, Theory of irreversible waves in Oscillographic polarography, J. Am. Chem. Soc. 75 (1953) 1190-1196, http://dx.doi.org/10.1021/ja01101a054.

[18] D.J. Schiffrin, Theory of cyclic voltammetry for reversible electrodeposition of in soluble products, J. Electroanal. Chem. 201 (1986) 199-203, http://dx.doi.org/10. 1016/0022-0728(86)90099-9.

[19] G. Mamantov, D.L. Manning, J.M. Dale, Reversible deposition of metals on solid electrodes by voltammetry with linearly varying potential, J. Electroanal. Chem. 9 (1965) 253-259, http://dx.doi.org/10.1016/0022-0728(65)80023-7.

[20] N. White, F. Lawson, Potential sweep voltammetry of metal deposition and dissolution: part I. Theoretical analysis, J. Electroanal. Chem. 25 (1970) 409-419, http://dx.doi.org/10.1016/S0022-0728(70)80102-4.

[21] F.H. Beyerlein, R.S. Nicholson, Influence of amalgam formation on cyclic voltam metry, Anal. Chem. 44 (1972) 1647-1650, http://dx.doi.org/10.1021/ ac60317a007.

[22] K. Tokuda, N. Enomoto, H. Matsuda, N. Koizumi, Cyclic voltammetry at hanging mercury drop electrodes for reversible amalgam formation and dissolution reactions, J. Electroanal. Chem. 159 (1983) 23-32, http://dx.doi.org/10.1016/S0022 0728(83)80311-8.

[23] S. Martinet, J. Bouteillon, J.P. Caire, Modelling of cyclic voltammograms for twostep metal deposition on an inert electrode with adsorption, J. Appl. Electrochem. 28 (1998) 819-825, http://dx.doi.org/10.1023/A:1003424123260.

[24] D. Krulic, N. Fatouros, D. Liu, A complementary survey of staircase voltammetry with metal ion deposition on macroelectrodes, J. Electroanal. Chem. 754 (2015) 30-39, http://dx.doi.org/10.1016/J.JELECHEM.2015.06.012.

[25] W. Zhou, Y.-L. Liu, K. Liu, Z.-R. Liu, L.-Y. Yuan, L. Wang, Y.-X. Feng, Z.-F. Chai, W.Q. Shi, Electroreduction of $\mathrm{Gd}^{3+}$ on $\mathrm{W}$ and $\mathrm{Zn}$ electrodes in $\mathrm{LiCl}-\mathrm{KCl}$ eutectic: a comparison study, J. Electrochem. Soc. 162 (2015) D531-D539, http://dx.doi.org/ $10.1149 / 2.0541510$ jes.

[26] H. Tang, B. Pesic, Electrochemical behavior of $\mathrm{LaCl}_{3}$ and morphology of La deposit on molybdenum substrate in molten $\mathrm{LiCl}-\mathrm{KCl}$ eutectic salt, Electrochim. Acta 119 (2014) 120-130, http://dx.doi.org/10.1016/J.ELECTACTA.2013.11.148.

[27] A. Samin, E. Wu, J. Zhang, The thermodynamic and transport properties of $\mathrm{GdCl}_{3}$ in molten eutectic LiCl-KCl derived from the analysis of cyclic voltammetry signals, J. Appl. Phys. 121 (2017) 74904, http://dx.doi.org/10.1063/1.4976570.

[28] D. Liu, D. Krulic, H. Groult, N. Fatouros, Silver ion deposition on gold and silver disc electrodes from aqueous solutions and from dry or wet [EMIM][NTf2] room-temperature ionic liquid, J. Electroanal. Chem. 775 (2016) 91-104, http://dx.doi.org/ 10.1016/J.JELECHEM.2016.05.004.
[29] P. Peljo, D. Lloyd, N. Doan, M. Majaneva, K. Kontturi, Towards a thermally regenerative all-copper redox flow battery, Phys. Chem. Chem. Phys. 16 (2014) 2831-2835, http://dx.doi.org/10.1039/C3CP54585G.

[30] Y. Li, J. Sniekers, J. Malaquias, X. Li, S. Schaltin, L. Stappers, K. Binnemans, J. Fransaer, I.F.J. Vankelecom, A non-aqueous all-copper redox flow battery with highly soluble active species, Electrochim. Acta 236 (2017) 116-121, http://dx.doi. org/10.1016/J.ELECTACTA.2017.03.039.

[31] E. Gileadi, The enigma of metal deposition, J. Electroanal. Chem. 660 (2011) 247-253, http://dx.doi.org/10.1016/J.JELECHEM.2011.01.025.

[32] E. Gileadi, Problems in interfacial electrochemistry that have been swept under the carpet, J. Solid State Electrochem. 15 (2011) 1359-1371, http://dx.doi.org/10. 1007/s10008-011-1344-5.

[33] E. Gileadi, Can an electrode reaction occur without electron transfer across the metal/solution interface? Chem. Phys. Lett. 393 (2004) 421-424, http://dx.doi. org/10.1016/J.CPLETT.2004.06.070.

[34] E. Gileadi, Charge and mass transfer across the metal/solution Interface, Isr. J. Chem. 48 (2008) 121-131, http://dx.doi.org/10.1560/IJC.48.3-4.121.

[35] W.R. Fawcett, C.A. Foss, Role of the solvent in the kinetics of heterogeneous elec tron and ion transfer reactions, Electrochim. Acta 36 (1991) 1767-1774, http://dx doi.org/10.1016/0013-4686(91)85043-7.

[36] W.R. Fawcett, C.A. Foss, On the nature of the transfer coefficient for ion transfer reactions, J. Electroanal. Chem. 250 (1988) 225-230, http://dx.doi.org/10.1016/ 0022-0728(88)80209-2.

[37] L.M.C. Pinto, E. Spohr, P. Quaino, E. Santos, W. Schmickler, Why silver deposition is so fast: solving the enigma of metal deposition, Angew. Chem. Int. Ed. 52 (2013) 7883-7885, http://dx.doi.org/10.1002/anie.201301998.

[38] N. Fatouros, D. Krulic, H. Groult, Linear sweep and staircase voltammetries for reversible deposition of metal ions on the same metal and on foreign substrates, $\mathrm{J}$ Electroanal. Chem. 625 (2009) 1-6, http://dx.doi.org/10.1016/J.JELECHEM.2008. 07.028.

[39] A. Saila, Etude des systemes electrochimiques quasi-reversibles par voltamperometrie a balayage lineaire et semi-integration. Applications aux comportements de rhenium et dysprosium en milieux de sels fondus, Univ. Badji Mokhtar, Annaba, 2010http://biblio.univ-annaba.dz/wp-content/uploads/2015/01/SAILAAbdelkader.pdf.

[40] R.R. Bessette, J.W. Olver, Measurement of diffusion coefficients for the reduction of copper(I) and (II) in acetonitrile, J. Electroanal. Chem. 21 (1969) 525-529, http:// dx.doi.org/10.1016/S0022-0728(69)80329-3. 